\title{
Neutralizing antibodies respond to a bivalent dengue DNA vaccine or/and a recombinant bivalent antigen
}

\author{
ZHI-SHAN ZHANG ${ }^{1}$, YU-WEI WENG ${ }^{2}$, HAI-LONG HUANG ${ }^{2}$, JIAN-MING ZHANG ${ }^{2}$ and YAN-SHENG YAN ${ }^{2}$ \\ ${ }^{1}$ Clinical Laboratory, Affiliated Quanzhou First Hospital of Fujian Medical University; \\ ${ }^{2}$ Fujian Center for Disease Control and Prevention, Fuzhou, Fujian 350001, P.R. China
}

Received November 22, 2013; Accepted June 10, 2014

DOI: $10.3892 / \mathrm{mmr} .2014 .2850$

\begin{abstract}
There is currently no effective vaccine to prevent dengue infection, despite the existence of multiple studies on potential methods of immunization. The aim of the present study was to explore the effect of DNA and/or recombinant protein on levels of neutralizing antibodies. For this purpose, envelope domain IIIs of dengue serotypes 1 and 2 (DEN-1/2) were spliced by a linker (Gly-Gly-Ser-Gly-Ser) ${ }_{3}$ and cloned into the prokaryotic expression plasmid pET30a (+) and eukaryotic vector pcDNA3.1 (+). The chimeric bivalent protein was expressed in Escherichia coli, and one-step purification by high-performance liquid chromatography was conducted. Protein expression levels of the DNA plasmid were tested in BHK-21 cells by indirect immunofluorescent assay. In order to explore a more effective immunization strategy and to develop neutralizing antibodies against the two serotypes, mice were inoculated with recombinant bivalent protein, the DNA vaccine, or the two given simultaneously. Presence of the specific antibodies was tested by ELISA and the presence of the neutralizing antibodies was determined by plaque reduction neutralization test. Results of the analysis indicated that the use of a combination of DNA and protein induced significantly higher titers of neutralizing antibodies against either DEN-1 or DEN-2 (1:64.0 and 1:76.1, respectively) compared with the DNA (1:24.7 and 1:26.9, DEN-1 and DEN-2, respectively) or the recombinant protein (1:34.9 and 1:45.3 in DEN-1 and DEN-2, respectively). The present study demonstrated that the combination of recombinant protein and DNA as an immunization strategy may be an effective method for the development of a vaccine to prevent dengue virus infection.
\end{abstract}

\footnotetext{
Correspondence to: Dr Yan-Sheng Yan, Fujian Center for Disease Control and Prevention, 76 Jintai Road, Fuzhou, Fujian 350001, P.R. China

E-mail: yanysh_fj@163.com
}

Key words: dengue vaccine, dengue virus, envelope protein, domain III, immunogenicity

\section{Introduction}

Dengue fever (DF), a mosquito-borne viral disease caused by the dengue virus, is one of the most common infectious diseases in tropical and subtropical regions. Patients infected with dengue virus exhibit various clinical symptoms with different levels of infection, from self-limited DF to life-threatening dengue hemorrhagic fever (DHF) and dengue shock syndrome (DSS) (1). Currently, there is no effective antiviral therapy for patients with DF, and the lack of effective mosquito control measures in dengue endemic areas maintains a high incidence of dengue infection. Developing dengue vaccines may provide a realistic approach for controlling dengue infections.

Dengue viruses are members of the family Flaviviridae and exist as four antigenically distinct serotypes (DEN-1-4) (2). This has been a major hindrance to vaccine development, as an individual who has experienced a single dengue virus infection develops neutralizing antibodies to that specific serotype, but remains susceptible to the other three serotypes of dengue virus. The cross-reactive, non-neutralizing antibody from the first dengue infection may bind to other serotypes of dengue virus, enhancing the uptake of virions into monocytic cell lines through interaction with cell surface immunoglobulin (Ig) receptors. This is a phenomenon known as antibody-dependent enhancement of infection (ADE) (3). The mechanism of ADE is considered as an important contributing factor to DHF and DSS (4). Therefore, an effective dengue vaccine must induce long-lasting and tetravalent type-specific neutralizing antibodies against all four serotypes. At present, there is no effective vaccine available, despite much research into methods of immunization for dengue infection. Early efforts to develop a dengue vaccine used the live attenuated virus $(5,6)$ and inactivated whole virion vaccination methods (7). Other approaches principally focused on recombinant strategies, including the use of chimeric viruses encoding an antigen gene (8-10), antigen-encoding plasmids (11-13) and recombinant subunit antigens produced by heterologous expression systems (14-18).

The envelope $(\mathrm{E})$ protein is the major structural protein of the dengue virus, and the majority of the recombinant vaccine strategies of previous studies have focused on it, due to its biological function of host cell surface receptor binding (19). Previous studies have demonstrated that the E protein is able to induce neutralizing antibodies and protective immunity $(14,20)$. Further crystallographic studies have indicated 
that the E proteins from dengue viruses and other flaviviruses contain three structural domains (I, II and III) $(21,22)$. Domain III (DIII) is an independent IgG-like folding domain (23) that contains multiple type- and subtype-specific neutralizing epitopes and host cell receptor recognition sites $(19,24-27)$. Furthermore, it has been reported that either recombinant fusion proteins $(15,17)$ containing an envelope or plasmid-encoding DIII gene $(11,12)$ were able to induce neutralizing antibodies in mice. It has also been demonstrated that the fusion DIII protein does not elicit cross-reactive antibodies to heterologous dengue virus serotypes (15).

In the present study, domain III of the E protein (defined as EDIII) of the dengue type 1 and 2 viruses were spliced with a flexible peptide linker (Gly-Gly-Ser-Gly-Ser) $)_{3}$. The fusion gene was then subcloned into either a prokaryotic expression plasmid pET30a (+) or a eukaryotic expression vector pcDNA3.1 (+), then expressed in Escherichia (E.) coli and mammalian BHK-21 cells. The three immunization strategies were examined using the bivalent DNA vaccine, the recombinant bivalent antigen, or a combination of the two to determine whether the level of neutralizing antibodies is increased following immunization with a combination of two vaccines compared with levels of antibodies following a single vaccination.

\section{Materials and methods}

Materials. Escherichia coli host strains DH5 $\alpha$ and BL21 (DE3) were purchased from Takara Biotechnology, Dalian, China. The expression plasmid pET30a (+) was obtained from Novagen (Darmstadt, Germany) and pcDNA3.1 (+) from Invitrogen Life Technologies (Carlsbad, CA, USA). DEN-2 virus-specific monoclonal antibody (mAb; 3H5) (catalog no. MAB8702) was from Chemicon (Temecula, CA, USA). The secondary antibody-enzyme conjugates (anti-mouse IgG-alkaline phosphatase, anti-mouse IgG-fluorescein isothiocyanate conjugate) were obtained from SouthernBiotech (Birmingham, AL, USA). The ion exchange column (DEAE-5PW, TSK-GEL ${ }^{\circledR}$ ) was purchased from Tosoh Bioscience (Tokyo, Japan) and the high-performance liquid chromatography (HPLC) system (Agilent 1100 Series HPLC Value system) was from Agilent Technologies, Inc. (Santa Clara, CA, USA).

The DEN-1 (Hawaii strain) and DEN-2 (NGC strain) were gifts from Dr Hui-Ming Luo (Center for Disease Control and Prevention, Guangdong, China). C6/36 mosquito cells and BHK-21 cells were obtained from Wuhan Culture Collection, (Wuhan, China). C6/36 cells were maintained in RPMI-1640 medium (Gibco, Grand Island, NY, USA) with $10 \%$ fetal bovine serum (FBS) in a humidified incubator with $5 \% \mathrm{CO}_{2}$ at $28^{\circ} \mathrm{C}$. BHK-21 cells were cultured in the same medium as $\mathrm{C} 6 / 36$ but incubated at $37^{\circ} \mathrm{C}$ in a humidified $5 \% \mathrm{CO}_{2}$ incubator. The QIAmp Viral RNA Mini Kit, One-Step RT-PCR kit and the EndoFree Plasmid Maxi kit were obtained from Qiagen (Hilden, Germany).

Construction of expression vectors. C6/36 cells were infected (MOI=0.01-0.1 PFU/cell) with either DEN-1 or DEN-2 virus. After 4 days, tissue culture supernatants underwent RNA extraction using the QIAmp Viral RNA Mini Kit. The genome RNAs were used as templates to obtain DEN-1 and DEN-2 EDIII gene fragments (designated as D1EDIII and D2EDIII) by one-step real-time polymerase chain reaction (RT-PCR). To facilitate head-to-tail fusion of two fragments, a linker region consisting of (Gly-Gly-Ser-Gly-Ser) 3 was introduced, taking advantage of the presence of a $\mathrm{BamHI}$ site in its sequence. The sequence of the linker was GGTGGCTCTGGATCTGGCGGTTCTGGATCC GGTGGCTCTGGATCT (the underlined sequence is the BamHI restriction site). The primers used for PCR amplification are presented in Table I. The PCR was performed as follows: denaturing at $94^{\circ} \mathrm{C}$ for $2 \mathrm{~min}, 35$ cycles (denaturation at $94^{\circ} \mathrm{C}$ for $30 \mathrm{sec}$, renaturation at $60^{\circ} \mathrm{C}$ for $30 \mathrm{sec}$ and extension at $72^{\circ} \mathrm{C}$ for $30 \mathrm{sec}$ ), and final extension at $72^{\circ} \mathrm{C}$ for $7 \mathrm{~min}$. Following PCR, $10 \mu \mathrm{l}$ PCR products were separated using agarose gel electrophoresis. The PCR generated two fragments that were $\sim 400 \mathrm{bp}$ in length, predicted to code aa290-420 [encompassing the DEN-1 EDIII (aa293-412) and the DEN-2 EDIII (aa298-400) (28)]. The fragment D1EDIII was purified and digested with $K p n I$ and $B a m H I$, then ligated into the corresponding KpnI and BamHI restriction sites of the expression vector pET30a (+), generating a recombinant plasmid that was labeled as pET30a-D1EDIII. To create the D1/D2EDIII chimeric bivalent gene, fragment D2EDIII was digested with BamHI and XhoI and inserted into the BamHI and XhoI sites of pET30a-D1EDIII. The resulting pET30a-D1/2EDIII recombinant plasmid was identified by restriction analysis and sequencing.

To construct the recombinant eukaryotic expression vector, pET30a-D1/2EDIII was digested with KpnI and XhoI to release the chimeric bivalent gene, D1/2EDIII. The fragment D1/2EDIII was then ligated into the $K p n I / X h o I$ restriction sites of pcDNA3.1 to generate pcDNA-D1/2EDIII. The recombinant clones were amplified in E. coli DH5 $\alpha$ and purified with the EndoFree Plasmid Maxi purification kit (Takara Biotechnology, Dalian, China).

Expression and purification of recombinant pro-D1/D2EDIII protein in E. coli. E. coli BL21 (DE3) were transformed with plasmid pET30a-D1/2EDIII and grown in lysogeny broth (LB) medium (Sigma-Aldrich, St Louis, MO, USA) containing $30 \mu \mathrm{g} / \mathrm{ml}$ kanamycin at $37^{\circ} \mathrm{C}$ until an optical density (OD) of 0.6 was reached. Then, $0.6 \mathrm{mM}$ isopropylthiogalactoside (IPTG) was added. After $4 \mathrm{~h}, 1 \mathrm{ml}$ of cells was harvested, lysed and analyzed by SDS-PAGE. The bacteria transformed with expression vector pET30a (+) were included as negative controls. To obtain large yields of recombinant protein, bacteria transfected with pET30a-D1/2EDIII were used to inoculate into 1 liter LB growth medium containing $30 \mu \mathrm{g} / \mathrm{ml}$ kanamycin (Sigma-Aldrich). When the stationary growth phase was reached, $0.6 \mathrm{mM}$ IPTG was added to induce expression for $4 \mathrm{~h}$. The cell pellet was obtained by centrifugation $(4,000 \mathrm{x} \mathrm{g}$ for $10 \mathrm{~min}$ ), resuspended in lysis buffer [50 mM Tris- $\mathrm{HCl}$ (pH 7.2), $20 \mathrm{mM}$ EDTA and $100 \mathrm{mM} \mathrm{NaCl}$ ] and then sonicated. Following centrifugation at $12,000 \mathrm{x}$ g for $10 \mathrm{~min}$, aliquots of the supernatant and the pellet were analyzed by SDS-PAGE to determine whether the recombinant protein was soluble.

The pellet containing the inclusion bodies was resuspended in solubilization buffer (20 mM Tris- $\mathrm{HCl}$ ( $\mathrm{pH}$ 8.0), 5 mM EDTA, $100 \mathrm{mM} \mathrm{NaCl}$ and $8 \mathrm{M}$ urea), dialyzed overnight in dialysis buffer I [10 mM phosphate buffer ( $\mathrm{pH}$ 6.0) and $4 \mathrm{M}$ urea] and filtered through 0.22- $\mu \mathrm{m}$-pore filter units (EMD Millipore, Billerica, MA, USA). The supernantant was applied to HPLC on a cation exchange column (TSKgel DEAE-5PW, Tosoh 
Table I. Primers used in the present study.

\begin{tabular}{ll} 
Primer & Sequence (5'-3') \\
\hline D1F & 5'-GTGGTACCATGGACAAACTGACCTTAAAAGGG-3' \\
D1R & 5'-GTGGATCCAGAACCGCCAGATCCAGAGCCACCCCATGCGGTGTCTCCTAG-3' \\
D2F & 5'-GTGGATCCGGTGGCTCTGGATCT GACAAACTACAGCTCAAA-3' \\
D2R & 5'-GATCTCGAGTCACCAGGCTGTGTCACCTAAAAT-3'
\end{tabular}

Primers D1F and D1R were used to produce the D1EDIII gene. D1F contained 2/3 sequences of 5'-terminal linker (underlined). Primers D2F and D2R were used to generate the D2EDIII fragment. D2F contained 1/3 sequences of 3'-terminal linker. Primer D2R contained a stop codon (italics) at the C-terminal. Restriction sites (KpnI in D1F; BamHI in D1R and D2F; XhoI in D2R and D2HIS) are presented in bold.

Bioscience) pre-equilibrated with buffer I. The column was washed with buffer I for $30 \mathrm{~min}$, then with a linear gradient from buffer I to buffer II (1 M NaCl in buffer I) for $60 \mathrm{~min}$ and finally washed with buffer II for $30 \mathrm{~min}$. The elution fractions were collected and analyzed by SDS-PAGE. The elution containing the recombinant protein (designated as pro-D1/D2EDIII) was dialyzed against phosphate-buffered saline (PBS) to remove urea, and then the total protein from the E. coli transformants was analyzed by $12 \%$ SDS-PAGE, followed by Coomassie blue staining (Sigma-Aldrich).

Western blot analysis. Purified pro-D1/D2EDIII protein was purified by $12 \%$ SDS-PAGE and transferred electrophoretically to a nitrocellulose membrane. The membrane was blocked overnight with blocking buffer (5\% non-fat dried milk in PBS; Sigma-Aldrich) and then incubated with primary antibody (either a serum from mouse polyclonal anti-DEN-1 EDIII or anti-DEN-2 EDIII antibody at 1:500 dilution in blocking buffer) at room temperature for $1 \mathrm{~h}$. The membrane was washed with PBST (1X PBS containing 0.1\% Tween 20) three times and reacted with alkaline phosphatase-conjugated goat anti-mouse $\operatorname{IgG}$ at 1:5,000 in blocking buffer for $1 \mathrm{~h}$ at room temperature. Following washing, the protein bands were detected with 5-bromo-4-chloro-3 indolyl phosphate toluidinium (BCIP) and nitroblue tetrazolium chloride (NBT) (Sigma-Aldrich).

Cell transfection and immunoflourescence assay (IFA). BHK-21 cells were transfected with plasmid pcDNA-D1/2EDIII using Lipofectamine 2000 reagent (Life Technologies, Carlsbad, CA, USA). Cells were cultured in RPMI-1640 medium supplemented with $10 \% \mathrm{FBS}$ without antibiotic at $37^{\circ} \mathrm{C}$ and $5 \% \mathrm{CO}_{2}$. One day prior to transfection, cells were trypsinized and plated at a density of $1 \times 10^{5}$ cells/well on six-well plates, so that they were $40-50 \%$ confluent on the day of transfection. A total of $2 \mu \mathrm{g}$ pcDNA-D1/2 EDIII or pcDNA3.1 (+) (as negative control) was used and transfection was performed according to the manufacturer's instructions. Cells were analyzed $48 \mathrm{~h}$ post-transfection by indirect IFA using either a mouse polyclonal antibody prepared against purified bacterial expressed pro-D1/D2EDIII protein or mAb 3H5. Briefly, cells were washed once with PBS, air dried, fixed with ice-cold acetone and incubated for $45 \mathrm{~min}$ at $37^{\circ} \mathrm{C}$ with either polyclonal anti-pro-D1/D2EDIII antibody (at 1:40 dilution in 0.01 M PBS) or 3H5 mAb (at 1:200 dilution in $0.01 \mathrm{M}$ PBS). Cells were washed with PBS three times, incubated for
30 min with fluorescein-conjugated goat anti-mouse $\operatorname{IgG}$ (at 1:50 dilution in 0.01 M PBS), and detected under a fluorescent microscope.

Mouse immunization and antibody detection. Groups of BALB/c mice (4-6 weeks old; Huafukang Biotechnology Ltd., Beijing, China) were immunized intraperitoneally with $50 \mu \mathrm{g}$ recombinant pro-D1/D2EDIII protein emulsified with Freund's complete adjuvant (PRO group), and/or $100 \mu \mathrm{g}$ pcDNA-D1/2EDIII (DNA group or PRO/DNA group) on day 0,14 and 28 . The mice were maintained under a light/dark cycle, at $25^{\circ} \mathrm{C}$. Negative control mice received PBS at similar time-points. Blood was obtained from the mice 10 days after the final injection and sera were collected and stored at $-70^{\circ} \mathrm{C}$ until use. The mice were sacrificed by cervical dislocation 28 days after the final injection. Animal experiments were reviewed and approved by the Fujian Medical Research Center of Animal Care and adhered to the guidelines of the Government of China.

The antibody titers of mouse sera were evaluated by an indirect ELISA approach. Each of the monovalent DEN-1 and DEN-2 EDIII proteins (pro-D1EDIII, pro-D2EDIII) were used as a capture antigen to assess serotype specificity of antisera. Following coating for $2 \mathrm{~h}$ at $37^{\circ} \mathrm{C}$ with pro-D1EDIII or pro-D2EDIII (100 ng in $100 \mu \mathrm{l} /$ well), 96-well microplates were blocked with $1 \%$ bovine serum albumin in PBS-Tween 20 for $2 \mathrm{~h}$ at $37^{\circ} \mathrm{C}$, washed three times with PBS-Tween 20 and incubated with serial two-fold dilutions of mouse sera for $30 \mathrm{~min}$ at $37^{\circ} \mathrm{C}$. The wells were then washed a further three times and incubated with peroxidase-conjugated goat anti-mouse IgG (1:5,000 dilution in blocking buffer) at $37^{\circ} \mathrm{C}$ for $30 \mathrm{~min}$. Following three washes, 3,3'-5,5'-tetramethly benzidine substrate (100 $\mu \mathrm{l} /$ well $)$ was added and the samples were incubated at $37^{\circ} \mathrm{C}$ for $15 \mathrm{~min}$. The reaction was stopped by adding $50 \mu \mathrm{l}$ of $1 \mathrm{M} \mathrm{H}_{2} \mathrm{SO}_{4}$ and the absorbance of each well was read at $450 \mathrm{~nm}$ using a microplate reader (GelDox XR; Bio-Rad Laboratories, Hercules, CA, USA).

Neutralizing antibody titers were determined by plaque reduction neutralization test (PRNT) using either DEN-1 or DEN-2 virus with BHK-21 cells, as previously described (29). The neutralization titer was defined as the maximal dilution yielding a $50 \%$ reduction in the number of plaque-forming units, expressed as the $\mathrm{PRNT}_{50}$ titer.

Statistical analysis. Logarithmic transformations of the reciprocal $\mathrm{PRNT}_{50}$ titers of the mice in each immunization group 
A

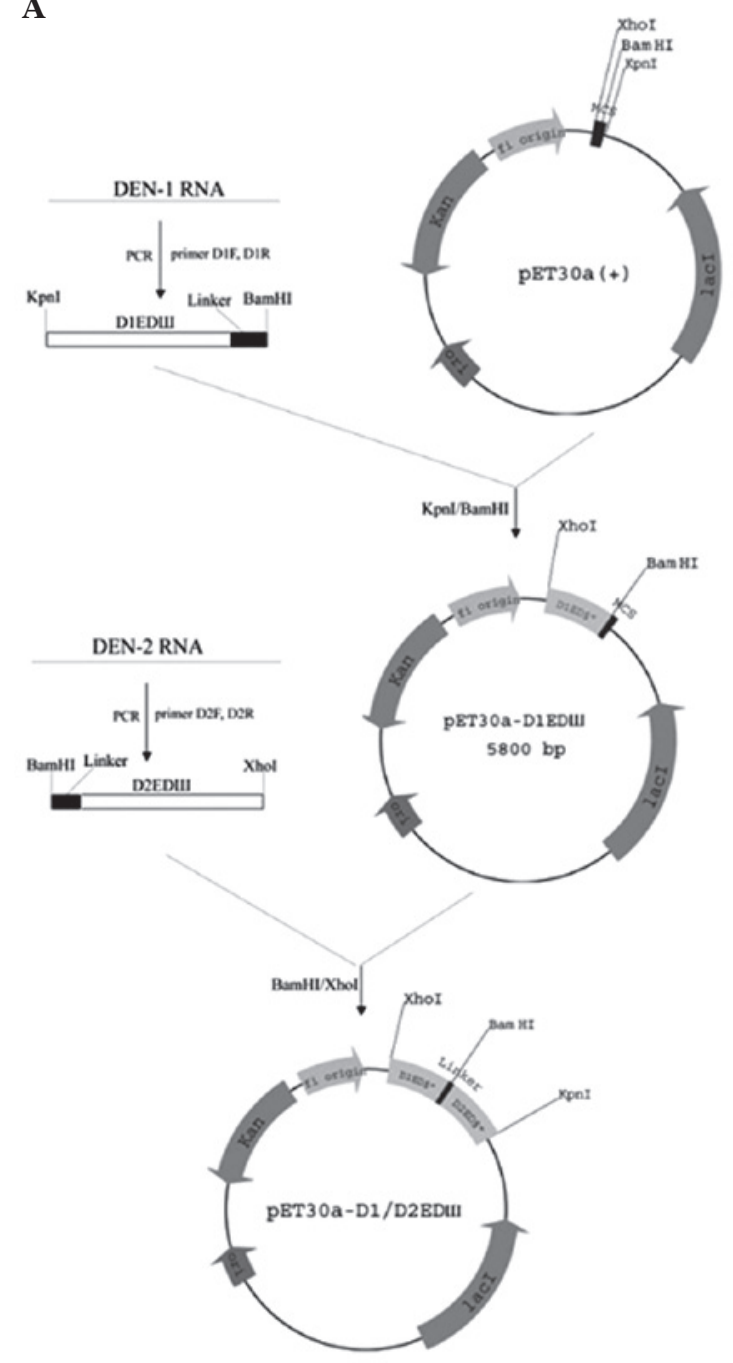

B
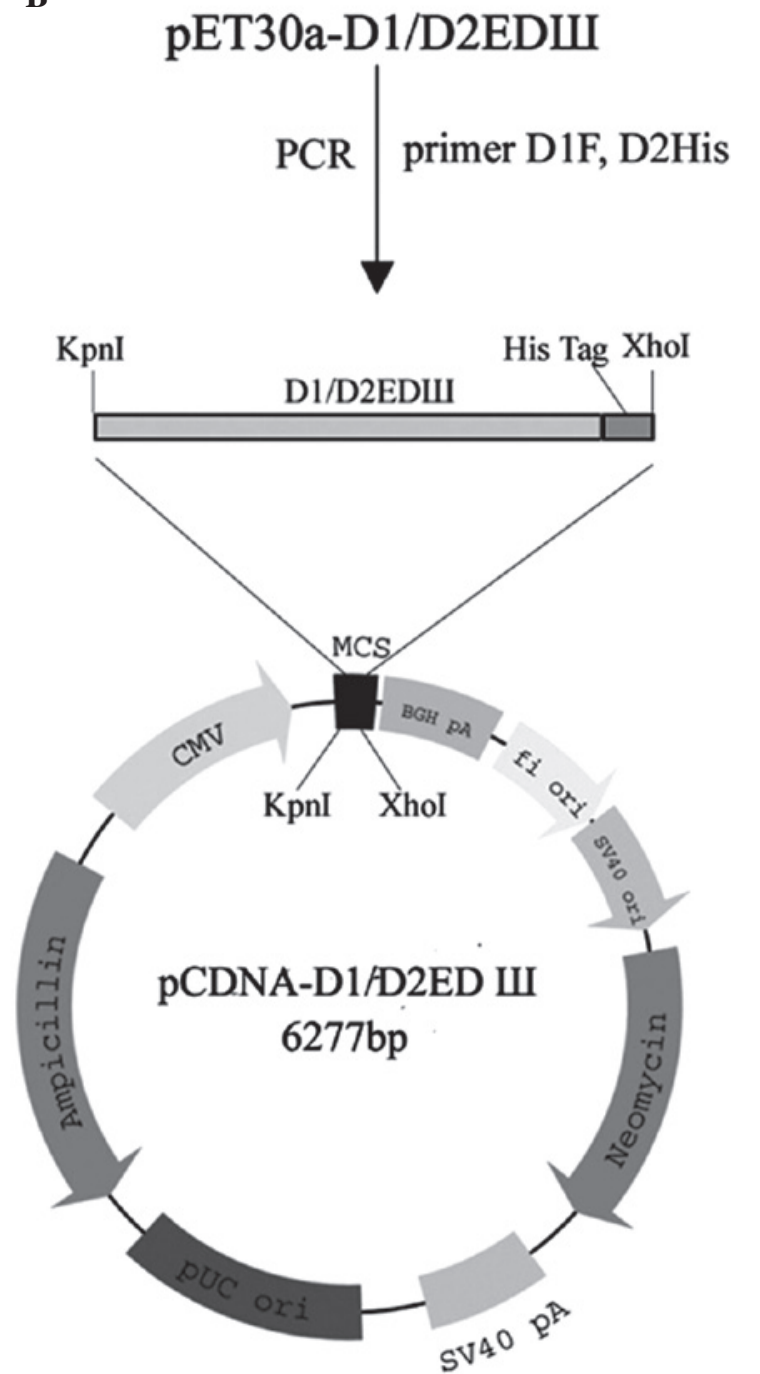

Figure 1. (A) Strategy for the construction of the recombinant prokaryotic expression plasmid pET30a-D1/2EDIII. The D1EDIII gene containing 2/3 sequence of linker at the C-terminus was inserted into the KpnI and BamHI sites of the E. coli expression plasmid pET30a (+) to generate the recombinant plasmid pET30a-D1EDIII. Next, the fragment D2EDIII containing 1/3 linker sequence at the N-terminus was subcloned into the BamHI and XhoI sites of pET30a-D1EDIII. In the resultant construct, pET30a-D1/2EDIII, the gene DEN-2 EDIII was fused in-frame to the 3' end of the DEN-1 EDIII, with a linker sequence between two genes. (B) Map of the recombinant eukaryotic expression vector pcDNA-D1/2EDIII. The bivalent gene D1/2EDIII with flanking KpnI and $\mathrm{XhoI}$ sites was obtained from pET30a-D1/2EDIII and inserted between the CMV promoter $(\mathrm{P} \mathrm{cmv})$ and the bovine growth hormone polyadenylation signal (BGH pA) of pcDNA3.1 (+). CMV, cytomegalovirus; DEN-1/2, dengue serotype 1/2.

were made, and the mean log titers were compared by analysis of variance. The mean log titers were converted to genometric mean (GM) titers for presentation. P-values were calculated using SAS version 8.2 statistical software (SAS Inc., Cary, NC, USA). $\mathrm{P}<0.05$ was considered to indicate a statistically significant difference.

\section{Results}

Creation of recombinant expression vectors. The construction of the recombinant pET30a-D1/2EDIII is illustrated in Fig. 1A. This construct was predicted to encode a 335-aa recombinant protein with a molecular weight of $37 \mathrm{kDa}$, and verified to be correct by restriction and sequencing (data not shown).

The D1/2EDIII chimeric bivalent gene was obtained from pET30a-D1/2EDIII by restriction digestion with KpnI/XhoI and cloned into the eukaryotic expression vector pcDNA3.1 (+) under the control of the cytomegalovirus promoter. A His tag was used to construct pcDNA-D1/2EDIII (Fig. 1B). The recombinant plasmid was correctly expressed in transfected BHK-21 cells, as demonstrated by IFA results (Fig. 2). The pcDNA-D1/2EDIII-transfected cells manifested a strong fluorescent signal when detected with either polyclonal anti-pro-D1/D2EDIII antibody or 3H5 mAb (Fig. 2A and C), compared with cells transfected with pcDNA3.1 (+) (Fig. 2Band D).

Expression and purification of recombinant pro-D1/D2EDIII bivalent protein in $E$. coli. The recombinant plasmid pET30a-D1/2EDIII was transformed into E. coli BL21 (DE3), and expression was induced by IPTG for $\sim 4 \mathrm{~h}$. Total protein from $E$. coli transformants was analyzed by $12 \%$ SDS-PAGE followed by Coomassie blue staining. As displayed in Fig. 3A, a dominant band corresponding to the predicted size was detected 

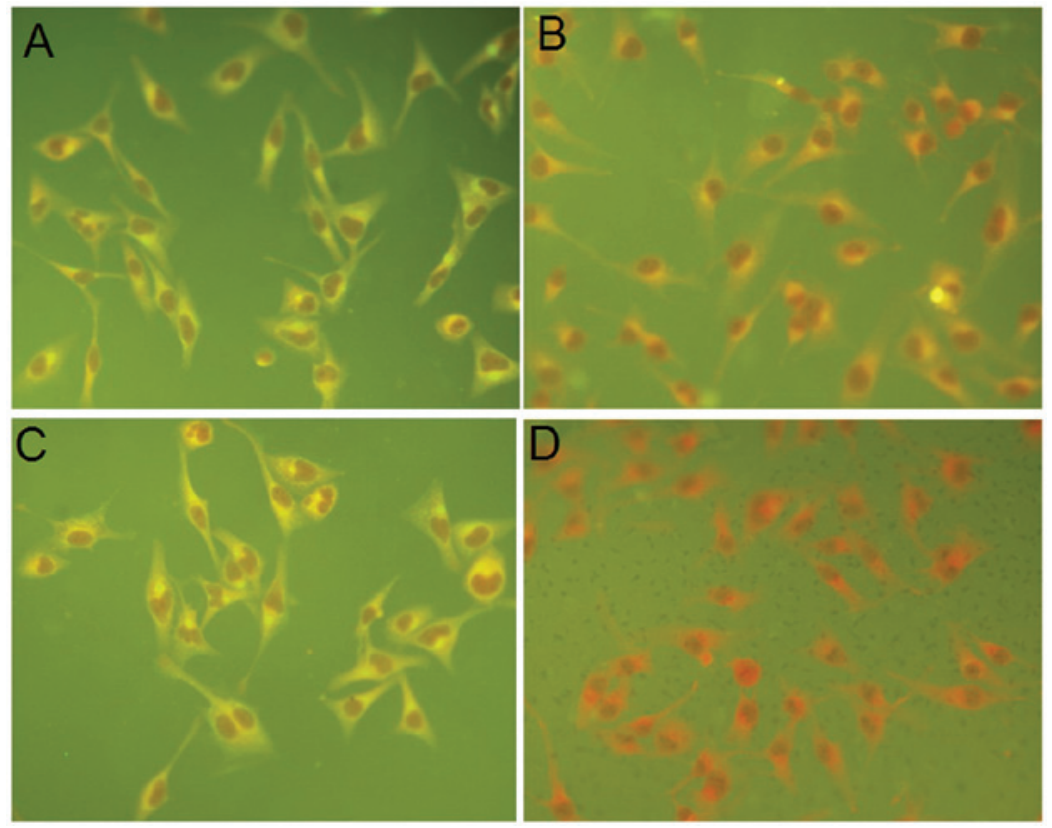

Figure 2. Detection of recombinant bivalent pro-D1/D2EDIII protein expression in BHK21 cells by fluorescence microscopy. BHK21 cells were transfected with (A and C) pcDNA-D1/2EDIII or (B and D) pcDNA3.1 (+). The cells were fixed with ice-cold acetone and analyzed by immunofluorescence assay using (A and B) polycolonal anti-pro-D1/D2EDIII antibody or (C and D) DEN-2-specific 3H5 monoclonal antibody in conjunction with a secondary fluorescein-conjugated goat anti-mouse immunoglobulin G. Magnification, x200.

A

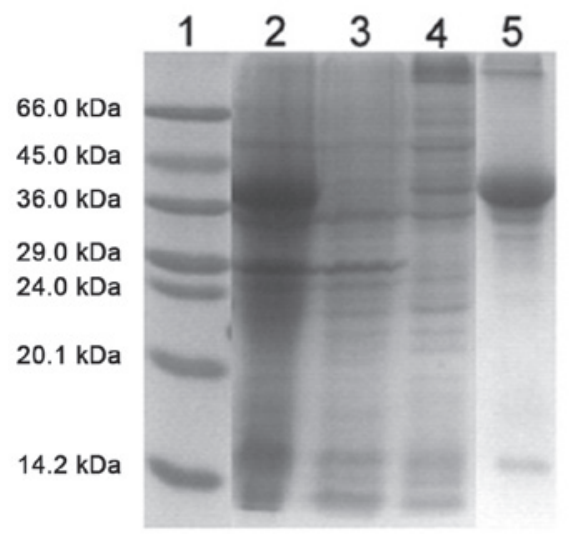

B

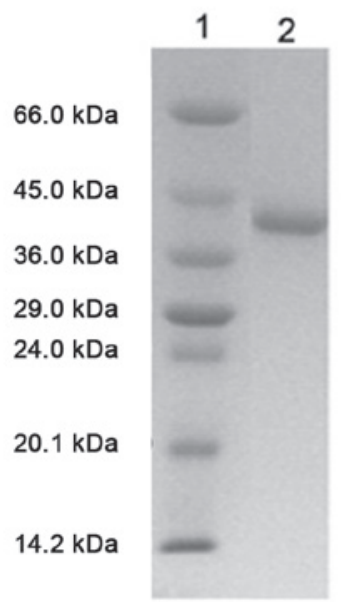

C

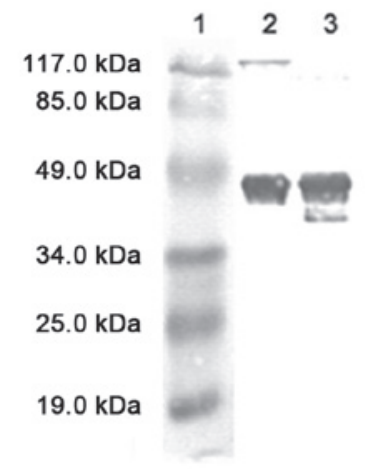

Figure 3. Expression and purification of recombinant bivalent pro-D1/D2EDIII protein. (A) Expression of recombinant bivalent pro-D1/D2EDIII protein in E. coli. Total protein from bacteria transformed with pET30a-D1/D2EDIII (lane 1) or with pET30a (lane 2) were separated using 12\% SDS-PAGE followed by Coomassie blue staining. Aliquots of supernatant (lane 4) and pellet (lane 5) of cell lysates from culture were analyzed also. Protein molecular weight markers were run in lane 1. Their sizes (in $\mathrm{kDa}$ ) are presented on the left. (B) SDS-PAGE analysis of purified pro-D1/DEDIII protein. Lane 1, protein molecular weight markers; and 2, purified pro-D1/DEDIII protein. (C) Western blot analysis of purified pro-D1/D2EDIII protein using mouse polyclonal anti-DEN-1 EDIII (lane 2) and anti-DEN-2 EDIII antibody (lane 3). Pre-stained protein molecular weight markers were run in lane 1, with their sizes displayed on the left. DEN-1/2, dengue serotype $1 / 2$.

in the pellet from bacteria carrying pET30a-D1/2EDIII (lane 2) but not from cells transformed with pET30a alone (lane 3). To examine the relative distribution of expressed recombinant protein in the soluble or insoluble fraction, 1 liter culture of bacteria transformed with pET30a-D1/2EDIII was prepared. The cell pellets were suspended in lysis buffer and sonicated. Following centrifugation, the supernatant and the pellet fractions were analyzed by SDS-PAGE. No dominant band was detected in the supernatant of the cell lysates (Fig. 3A, lane 4), but the majority of the expressed recombinant protein was associated with the pellet fraction (Fig. 3A, lane 5). Hence, the recombinant protein was present in the form of inclusion bodies.

The insoluble inclusion bodies were lysed in $8 \mathrm{M}$ urea, dialyzed in buffer I and applied to an HPLC cation exchange column. The fractions, eluted with varying concentrations of $\mathrm{NaCl}$ for different time periods, were colleted and analyzed by SDS-PAGE (data not shown), which indicated that the $37 \mathrm{kDa}$ pro-D1/2EDIII protein was eluted at $44-46 \mathrm{~min}$. The 44-46 min fractions were pooled and dialyzed to eliminate urea, and thus, purified D1/2EDIII protein was obtained. As demonstrated in Fig. 3B, lane 2, a single band of $37 \mathrm{kDa}$ was 
A

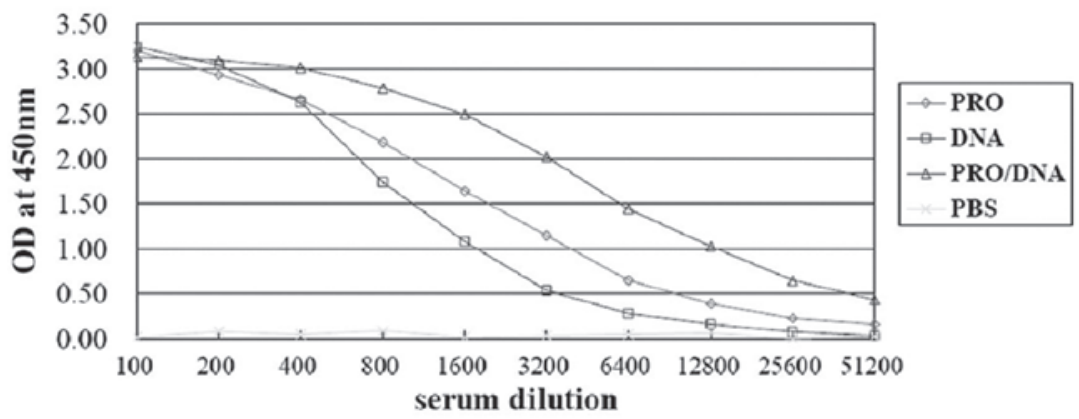

B

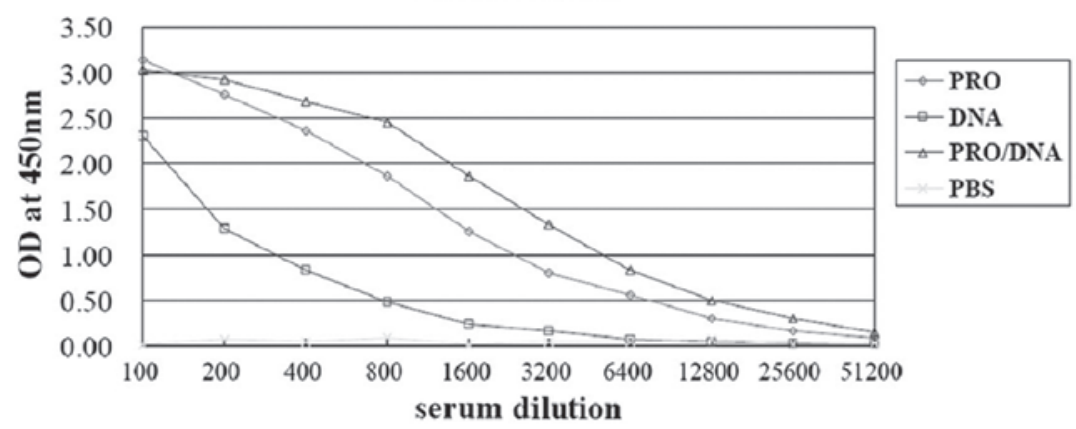

Figure 4. Determination of antibody titers in sera of immunized mice. Sera of mice immunized with the recombinant protein (PRO), the DNA vaccine (DNA), the combination of two vaccines (PRO/DNA) or phosphate-buffered saline were two-fold diluted and detected by ELISA using either (A) pro-D1EDIII or (B) pro-D2EDIII as a capture antigen. OD, optical density.

obtained following the purification steps, the final concentration of the purified protein was $1.05 \mathrm{mg} / \mathrm{ml}$. Thus, $20 \mathrm{mg}$ of purified protein was obtained from a liter culture of induced cells.

In order to demonstrate that the recombinant bivalent protein preserves the antigenicity of DEN-1 and DEN-2 EDIII, purified pro-D1/D2EDIII was detected by western blot assay using sera from mouse polyclonal anti-DEN-1 EDIII or anti-DEN-2 EDIII antibodies. The results are presented in Fig. 3C. The anti-DEN-1 EDIII or anti-DEN-2 EDIII antibody was able to recognize the chimeric pro-D1/2EDIII protein (lanes 2 and 3).

Serum antibody response in mice. All of the mice inoculated with recombinant protein or/and DNA demonstrated an IgG antibody response against either pro-D1EDIII (Fig. 4A) or pro-D2EDIII (Fig. 4B). The OD value of the PRO/DNA group was the highest and the D group was the lowest, while the PRO group exhibited an intermediate response. PBS-immunized sera did not display significant ELISA reactivity against pro-D1EDIII or pro-D2EDIII.

To evaluate the ability of antibodies to neutralize the DEN-1 and DEN-2 viruses, PRNT was performed. The results are summarized in Table II. The GM PRNT ${ }_{50}$ titers of the PRO/DNA group against either DEN-1 or DEN-2 were significantly higher than those of the PRO group $(\mathrm{P}<0.05)$, which were significantly higher than those of the DNA group $(\mathrm{P}<0.05)$.

\section{Discussion}

The development of a dengue vaccine continues to be a challenge, as the vaccine must protect against four serotypes of dengue virus without eliciting the ADE reaction in
Table II. Virus-neutralizing antibody titers in sera of immunized mice.

$\mathrm{GM} \mathrm{PRNT}_{50}$ titers

\begin{tabular}{lcc}
\cline { 2 - 3 } Immunogen & DEN-1 & DEN-2 \\
\hline PRO $(n=8)$ & 34.9 & 45.3 \\
DNA $(n=8)$ & 24.7 & 26.9 \\
PRO/DNA $(n=8)$ & 64.0 & 76.1 \\
PBS $(n=8)$ & ND & ND \\
\hline
\end{tabular}

PRO, recombinant protein; DNA, DNA vaccine; PRO/DNA, combination of PRO and DNA; PBS, mock immunized with phosphate-buffered saline; GM, geometric mean; ND, no detectable neutralizing antibody titer; PRNT, plaque reduction neutralization test; DEN-1/2, dengue serotype $1 / 2$.

order to be effective. Previous approaches have focused on live attenuated dengue virus, and development of a 'tetravalent' formulation by mixture of four monovalent dengue serotypes (5,30-34). However, simple physical mixture may lead to a predominant immune response towards a single serotype of dengue virus, which has been attributed to viral interference $(8,33,35,36)$. Recently, increasing attention is being focused on the recombinant strategies, mainly based on subunit proteins or DNA plasmids.

The EDIII of the DEN-2 virus has been successfully expressed in $E$. coli with or without fusion protein $(15,17,37)$, and these investigations demonstrated that the recombinant DEN-2 EDIII protein has the biological function of blocking the virus from binding to host cells (37) and the ability to 
elicit a neutralizing antibody reaction $(15,17)$. A tetravalent dengue vaccine, created by physically mixing four monovalent components into a single formulation based on domain III, has also been studied $(12,16)$. However, a single tetravalent vaccine has a clear advantage over a mixture of four 'monovalent' components, as it provides a significant cost benefit and has the potential to eliminate non-uniform immune responses.

In the present study, a bivalent dengue subunit vaccine and DNA vaccine were constructed using a prokaryotic expression plasmid or eukaryotic expression vector containing a bivalent gene, obtained by splicing EDIIIs of dengue virus serotypes 1 and 2 . A recombinant plasmid pET30a-D1/2EDIII was created by splicing the EDIII genes of DEN-1 and 2. A linker (Gly-Gly-Ser-Gly-Ser) ${ }_{3}$ was introduced to maintain structure integrity and biological function of the two serotype-specific EDIII proteins, taking advantage of glycine's lack of a $\beta$ carbon and serine's propensity of hydrogen bonding (38). The recombinant bivalent antigen was successfully expressed in $E$. coli and purified by HPLC with a yield of $\sim 20 \mathrm{mg} /$ liter. To verify whether the purified pro-D1/D2EDIII protein still retained the antigenicity of the monovalent EDIIIs it was derived from, a western blot analysis was performed using mouse polyclonal anti-DEN-1 or DEN-2 EDIII antibodies. The results demonstrated that the recombinant bivalent protein was recognized by the anti-DEN-1 or DEN-2 EDIII antibodies. Furthermore, the result of the ELISA indicated that the antibodies from mice immunized with the recombinant protein may react with monovalent pro-D1EDIII and D2EDIII at high levels. It is possible that the recombinant bivalent protein preserved the antigenic integrity of its precursors.

Furthermore, it was determined whether these antibodies were able to neutralize the DEN-1 and DEN-2 viruses. The PRNT data indicated that the GM PRNT ${ }_{50}$ titers of antibody were 34.9 for DEN-1 and 45.3 for DEN-2. Neutralizing antibody titers are widely accepted as markers of protective immunity, with $\mathrm{PRNT}_{50}$ titers of 1:10 being considered indicative of protective immunity $(39,40)$. The present study suggested that the recombinant bivalent protein was able to produce protective antibodies against the DEN-1 and DEN-2 viruses. These results are similar to those obtained by Khanam et al (41), who created a chimeric dengue antigen expressed in E. coli by splicing EDIIIs of virus serotypes 2 and 4 that was able to elicit neutralizing antibodies against dengue virus serotypes 2 and 4 . The titers of neutralizing antibodies have been demonstrated to be lower than those induced by EDIII-MBP proteins (16). The contrast is theorized to be attributed to the differences of the experimental parameters of the PRNT assays and the host cells that were used. Furthermore, the uncertainty of the carrier activity and safety of MBP in humans causes an obstacle in the application of these fusion proteins in a vaccine.

Promising DNA vaccines have been developed against the dengue virus previously: Mota et al (12) developed a tetravalent DNA vaccine consisting of a mixture of four plasmids, each encoding EDIII genes specific to one DEN serotype. However, extrapolation from the study by Mota et al (12) was limited as only the level of DEN-2 neutralizing antibodies was examined and the other three serotypes were not evaluated. In the present study, a pcDNA-D1/2EDIII recombinant plasmid was generated, which, as a bivalent gene, encoded the EDIIIs of DEN-1 and 2. To develop an effective DNA vaccine, it is essential that the recombinant protein, encoded by the DNA plasmid, is expressed correctly (42). The result of IFA indicated that cells transfected with pcDNA-D1/2EDIII were able to react with anti-pro-D1/D2EDIII antibody (Fig. 2A) or the $3 \mathrm{H} 5 \mathrm{mAb}$ (Fig. 2C), which is specific to DEN-2 EDIII $(29,30)$. The results of the present study suggested that the recombinant plasmid pcDNA-D1/2EDIII was capable of being expressed adequately in mammalian cells. The GM $\mathrm{PRNT}_{50}$ titers induced by DNA were 24.7 for DEN-1 and 26.9 for DEN-2, which is in contrast with the neutralizing antibody level of 1:10 that was reported by Mota et al (12).

It has previously been reported that the use of a combination of DNA vaccine and recombinant protein with a prime-boost strategy can induce higher antibody titers than the individual vaccines alone $(11,43,44)$. A study by Simmons et al (11) indicated that the combination of a DEN-2 DNA vaccine coding for premembrane and $E$ proteins and a recombinant fusion protein containing the domain III of the DEN-2 E protein fused to the maltose-binding protein (MBP) induced higher neutralizing titers than the DNA or protein alone. In the current study, various immunization strategies were tested to observe whether it is possible to increase the level of neutralizing antibodies against DEN-1 and DEN-2 induced by a combination of DNA and protein, compared with when the vaccines were used alone. The results indicated that when mice were immunized with a combination of DNA and protein, significantly higher titers of neutralizing antibodies against either DEN-1 or DEN-2 (64.0 or 76.1, respectively) were observed, compared with titers following vaccination with the DNA or the recombinant protein alone. Similar antibody responses were observed using ELISA, and an association between total antibody levels measured by ELISA and neutralizing antibody titers was indicated. This result is in contrast with the study by Simmons et al (11), which noted a lack of correlation between total antibody levels and $\mathrm{PRNT}_{50}$ titers. This contrast may be explained by the fact that Simmon et al (11) had a larger number of total epitopes available in the DNA vaccine coding for the premenbrane and E proteins, whereas the present study used only domain IIIs for the construction of a DNA vaccine.

In conclusion, a recombinant prokaryotic/eukaryotic expression plasmid containing fusion EDIIIs of DEN-1 and DEN-2 was successfully constructed in the present study, using a flexible peptide linker. The recombinant protein and DNA vaccines were able to induce neutralizing antibodies against DEN serotypes 1 and 2. The current strategies used to construct fusion bivalent genes, expression and one-step purification are valuable in developing an inexpensive scale-up tetravalent dengue subunit vaccine or DNA vaccine. However, the combination of bivalent DNA and the recombinant protein resulted in higher levels of neutralizing antibodies, and may have the potential to induce improved cell-mediated immune responses in addition to humoral immune responses, compared with DNA or protein subunit vaccines alone. Further studies are required to assess the ability to release cytokines and the duration of neutralizing antibodies in response to the three immunization strategies (DNA vaccine, recombinant subunit vaccine, or a combination of the two). 


\section{References}

1. Agarwal R, Kapoor S, Nagar R, et al: A clinical study of the patients with dengue hemorrhagic fever during the epidemic of 1996 at Lucknow, India. Southeast Asian J Trop Med Public Health 30: 735-740, 1999.

2. Rosen L: Comments on the epidemiology, pathogenesis and control of dengue. Med Trop (Mars) 59: 495-498, 1999.

3. Morens DM and Halstead SB: Measurement of antibody-dependent infection enhancement of four dengue virus serotypes by monoclonal and polyclonal antibodies. J Gen Virol 71: 2909-2914, 1990.

4. Anderson R, Wang S, Osiowy C and Issekutz AC: Activation of endothelial cells via antibody-enhanced dengue virus infection of peripheral blood monocytes. J Virol 71: 4226-4232, 1997.

5. Bhamarapravati $N$ and Sutee Y: Live attenuated tetravalent dengue vaccine. Vaccine 18 (Suppl 2): 44-47, 2000.

6. Eckles KH, Dubios DR, Putnak R, et al: Modification of dengue virus strains by passage in primary dog kidney cells: preparation of candidate vaccines and immunization of monkeys. Am J Trop Med Hyg 69 (Suppl): 12-16, 2003.

7. Putnak R, Barvir DA, Burrous JM, et al: Development of a purified, inactivated, dengue-2 virus vaccine prototype in Vero cells: immunogenicity and protection in mice and rhesus monkeys. J Infect Dis 174: 1176-1184, 1996.

8. Guirakhoo F, Pugachev K, Zhang Z, et al: Safety and efficacy of chimeric yellow fever-dengue virus tetravalent vaccine formulations in nonhuman primates. J Virol 78: 4761-4775, 2004

9. Van der Most RG, Murali-Krishna K, Ahmed R and Strauss JH Chimeric yellow fever/dengue virus as a candidate dengue vaccine: Quantitation of the dengue virus-specific CD8 T-cell response. J Virol 74: 8094-8101, 2000.

10. Guirakhoo F, Arroyol J, Pugachev KV, et al: Construction, safety, and immunogenecity in nonhuman primates of a chimeric yellow fever dengue virus tetravalent vaccine. J Virol 75: 7290-7304, 2001.

11. Simmons M, Murphy GS, Kochel T, et al: Characterization of antibody responses to combinations of a dengue-2 DNA and dengue-2 recombinant subunit vaccine. Am J Trop Med Hyg 65: 420-426, 2001

12. Mota J, Acosta M, Argotte R et al: Induction of protective antibodies against dengue virus by tetravalent DNA immunization of mice with domain III of the envelope protein. Vaccine 23 : 3469-3476, 2005.

13. Wu SF, Liao CL, Lin YL, et al: Evaluation of protective efficacy and immune mechanisms of using a non-structural protein NS1 in DNA vaccine against dengue 2 virus in mice. Vaccine 21 : 3919-3929, 2003.

14. Men R, Wyatt L, Tokimatsu I, et al: Immunization of rhesus monkeys with a recombinant of modified vaccina virus Ankara expressing a truncated envelope glycoprotein of dengue type 2 virus induced resistance to dengue type 2 virus challenge. Vaccine 18: 3113-3122, 2000

15. Simmons M, Nelson WM, Wu SJ and Hayes CG: Evaluation of the protective efficacy of a recombinant dengue envelope $B$ domain fusion protein against dengue 2 virus infection in mice. Am J Trop Med Hyg 58: 655-662, 1998.

16. Simmons M, Murphy GS and Hayes CG: Short report: Antibody response of mice immunized with a tetravalent dengue recombinant protein subunit vaccine. Am J Trop Med Hyg 65: 159-161, 2001.

17. Hermida L, Rodriguez R, Lazo L, et al: A dengue-2 envelope fragment inserted within the structure of the P64k meningococcal protein carrier enables a functional immune response against the virus in mice. J Virol Methods 115: 41-49, 2004.

18. Kelly EP, Greene JJ, King AD and Innis BL: Purified dengue 2 virus envelope glycoprotein aggregates produced by baculovirus are immunogenic in mice. Vaccine 18: 2549-2559, 2000.

19. Chen Y, Maguire T and Marks RM: Demonstration of binding of dengue envelope protein to target cells. J Virol 70: 8765-8772, 1996.

20. Bray M and Lai CJ: Dengue virus premembrane and membrane proteins elicit a protective immune response. Virology 185: 505-508, 1991

21. Rey FA, HeinA FX, Mandl C, et al: The envelope glycoprotein from tick-borne encephalitis virus at 2 A resolution. Nature 375: 291-298, 1995.

22. Roehrig JT, Volpe KE, Squires J, et al: Contribution of disulfide bridging to epitope expression of the dengue type 2 virus envelope glycoprotein. J Virol 78: 2648-2652, 2004.
23. Bhardwaj S, Holbrook M, Shope RE, et al: Biophysical characterization and vector-specific antagonist activity of domain III of the tick-borne flavivirus envelope protein. J Virol 75: 4002-4007, 2001.

24. Crill WD and Roehrig RT: Monoclonal antibodies that bind to domain III of dengue virus E glycoprotein are the most efficient blockers of virus E glycoprotein are most efficient blockers of virus adsorption to Vero cells. J Virol 75: 7769-7773, 2001

25. Megret F, Hugnot JP, Falconar MK, et al: Use of recombinant fusion proteins and monoclonal antibodies to define linear and discontinuous antigenic sites on the dengue envelope glycoprotein. Virology 187: 480-491, 1992.

26. Roehrig JT, Johnson AJ, Hunt AR, et al: Antibodies to dengue 2 virus E-glycoprotein synthetic peptides identity antigenic conformation. Virology 177: 668-675, 1990.

27. Hung JJ, Hsieh MT, Young MJ, et al: An external loop region of domain III of dengue virus type 2 envelope protein is involved in serotype-specific binding to mosquito but not mammalian cells. J Virol 78: 378-388, 2004

28. Simmons M, Nelson WM, Wu SJ and Hayes CG: Evaluation of the protective efficacy of a recombinant dengue envelope $B$ domain fusion protein against dengue 2 virus infection in mice. Am J Trop Med Hyg 58: 655-662, 1998.

29. Morens DM, Halstead SB, Repik PM, et al: Simplified plaque reduction assay for dengue viruses by semi-micromethods in BHK21 cells: comparison of the BHK suspension test standard plaque reduction neutralization. J Clin Microbio 22: 250-254, 1985.

30. Trirawatanapong T, Chandran B, Putnak R and Padmanabhan R: Mapping of a region of dengue virus type- 2 glycoprotein required for binding by a neutralizing monoclonal antibody. Gene 116: 139-150, 1992 .

31. Sun W, Edelman R, Kanesa-Thasan N, et al: Vaccination of human volunteers with monovalent and tetravalent live-attenuated dengue vaccine candidates. Am J Trop Med Hyg 69 (Suppl): 24-31, 2003.

32. Edelman R, Wasserman SS, Bodison SA, et al: Phase I trial of 16 formulations of a tetravalent live-attenuated dengue vaccine. Am J Trop Med Hyg 69 (Suppl): 48-60, 2003.

33. Kitchener S, Nissen M, Nasveld P, et al: Immunogenicity and safety of two live-attenuated tetravalent dengue virus vaccine formulations in healthy Australian adults. Vaccine 24: 1238-1241, 2006.

34. Sabchareon A, Lang J, Chanthavanich P, et al: Safety and immunogenicity of a three dose regimen of two tetravalent live-attenuated dengue vaccines in five- to twelve-year-old Thai children. Pediatr Infect Dis J 23: 99-109, 2004.

35. Swaminathan $\mathrm{S}$ and Khanna N: Viral vaccines for dengue: the present and the future. WHO Dengue Bul 27: 181-191, 2003.

36. Kanesa-thasan N, Sun W, Kim-Ahn G, et al: Safety and immunogenicity of attenuated dengue virus vaccines (Aventis Pasteur) in human volunteers. Vaccine 19: 3179-3188, 2001.

37. Jaiswal S, Khanna N and Swaminathan S: High-level expression and one-step purification of recombinant dengue virus type 2 envelope domain III protein in Escherichia coli. Protein Expr Purif 33: 80-91, 2004.

38. Robinson CR and Sauer RT: Optimizing the stability of single-chain proteins by linker length and composition mutagenesis. Pro Natl Acad Sci USA 95: 5929-5934, 1998.

39. Putnak R, Feighny R, Burrous J, et al: Dengue-1 virus envelope glycoprotien gene expressed in recombinant baculovirus elicits virus-neutralizing antibody in mice and protects them from virus challenge. Am J Trop Med Hyg 45: 159-167, 1991.

40. Delenda C, Staropoli I, Frenkiel MP, et al: Analysis of C-terminally truncated dengue 2 and dengue 3 virus envelope glycoproteins: processing in insect cells and immunogenic properties in mice. J Gen Virol 75: 1569-1578, 1994

41. Khanam S, Etemad B, Khanna N and Swaminathan S: Induction of neutralizing antibodies specific to dengue virus serotypes 2 and 4 by a bivalent antigen composed of linked envelope domains III of these two serotypes. Am J Trop Med Hyg 74: 266-277, 2006.

42. Chang GJ, Davis BS, Hunt AR, et al: Flavivirus DNA vaccines: current status and potential. Ann N Y Acad Sci 951: 272-285, 2001.

43. Sedegah M, Jones TR, Kaur M, et al: Boosting with recombinant vaccinia increases immunogenicity and protective efficacy of malaria DNA vaccine. Proc Natl Acad Sci USA 95: 7648-7653, 1998.

44. Barnett SW, Rajasekar S, Legg H, et al: Vaccination with HIV-1 gp120 DNA induces immune responses that are boosted by a recombinant gp 120 protein subunit. Vaccine 15: 869-873, 1997. 